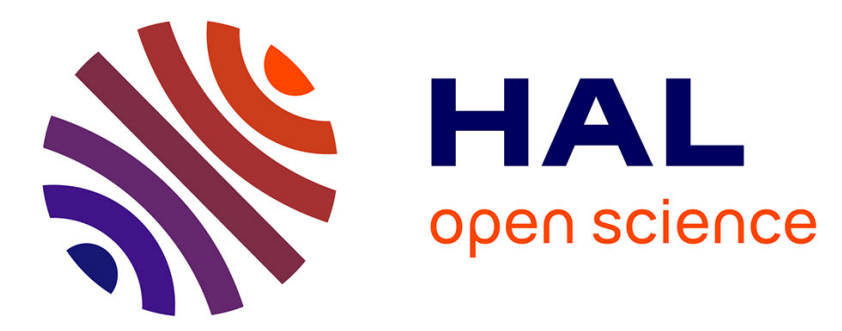

\title{
Diversity techniques for a free-space optical communication system in correlated log-normal channels
}

\author{
Mohamed Abaza, Raed Mesleh, Ali Mansour, El-Hadi Aggoune
}

\section{To cite this version:}

Mohamed Abaza, Raed Mesleh, Ali Mansour, El-Hadi Aggoune. Diversity techniques for a free-space optical communication system in correlated log-normal channels. Optical Engineering, 2014, 53 (1), pp.1-6. 10.1117/1.OE.53.1.016102 . hal-00923801

\section{HAL Id: hal-00923801 \\ https://hal.univ-brest.fr/hal-00923801}

Submitted on 4 Jan 2014

HAL is a multi-disciplinary open access archive for the deposit and dissemination of scientific research documents, whether they are published or not. The documents may come from teaching and research institutions in France or abroad, or from public or private research centers.
L'archive ouverte pluridisciplinaire HAL, est destinée au dépôt et à la diffusion de documents scientifiques de niveau recherche, publiés ou non, émanant des établissements d'enseignement et de recherche français ou étrangers, des laboratoires publics ou privés. 


\title{
Diversity techniques for a free-space optical communication system in correlated log-normal channels
}

\author{
Mohamed Abaza, ${ }^{a, \star}$ Raed Mesleh, ${ }^{a}$ Ali Mansour, ${ }^{b}$ and El-Hadi M. Aggoune ${ }^{a}$ \\ aUniversity of Tabuk, Sensor Networks and Cellular System Research Center, Electrical Engineering Department, 71491 Tabuk, Saudi Arabia \\ bENSTA Bretagne, Lab STICC, 2 Rue François Verny, 29806 Brest Cedex, France
}

\begin{abstract}
Performance analysis of free-space optical (FSO) communication systems in different channel conditions has gained significant attention in literature. Nevertheless, most existing studies consider uncorrelated channel conditions. An uncorrelated channel requires sufficient spacing between transmitters and limits the receiver field of view and link distance. However, this might not be feasible in all applications. Thereby, this paper studies repetition code (RC) and orthogonal space time block code (OSTBC) performance in correlated log-normal FSO channels using intensity modulation and direct detection. An approximate analytical expressions using moment generating function for the average bit error probability are derived. Our simulation results show that RCs are superior to OSTBCs in correlated channel conditions. @ 2014 Society of Photo-Optical Instrumentation Engineers (SPIE) [DOI: 10.1117/1.OE.53.1.XXXXXX]
\end{abstract}

Keywords: free-space optical communications; atmospheric turbulence; spatial diversity; orthogonal space time block codes; repetition codes.

Paper 131244 received Aug. 14, 2013; revised manuscript received Nov. 16, 2013; accepted for publication Dec. 4, 2013.

\section{Introduction}

Free-space optical (FSO) technology has several advantages compared with radio frequency $(\mathrm{RF})$, such as high data rates, license-free operation, high security, and ease of deployment. However, the performance of FSO systems can be highly affected by fog and atmospheric turbulence. ${ }^{1-3}$ Atmospheric turbulence can be considered as one of the main problems facing FSO communication systems. The nonhomogeneous changes in pressure and temperature lead to several changes in the refractive index, which result in rapid fluctuations in the received signal and limit the performance of the FSO communication system. . $^{1,2,5}$

Spatial diversity has the ability to mitigate the degradation of the bit error rate (BER) caused by atmospheric turbulence and maximizes the data rate of the system. Furthermore, it can improve the link reliability, decreases the blockage probability, and limits the need for active tracking due to laser misalignment. ${ }^{1,4,6-10}$ Two codes are generally considered in FSO spatial diversity systems. Namely, repetition codes (RCs) and orthogonal space time block codes (OSTBCs).

In the RC system, the same signal is transmitted simultaneously from the available transmitters. The advantages of such a transmission technique are very limited in RF and FSO wireless systems using a heterodyne (coherent) reception. However, heterodyne reception is not preferred in FSO systems due to its high cost and complexity. An alternative and preferred transmission/reception method is intensity modulation with direct detection (IM/DD). ${ }^{11} \mathrm{~A}$ study in Ref. 2 considers the IM/DD RCs system with on-off keying (OOK) modulation over FSO log-normal channels. Reported results ${ }^{2}$ demonstrate that significant performance gains can be achieved.

A modified version of Alamouti's code, which allows the use of unipolar pulsed modulation techniques for IM/DD

*Address all correspondence to: Mohamed Abaza, E-mail: mabaza.sncs@ut .edu.sa links is reported in Ref. 12. The main idea is to represent the negative signal of a unipolar signal by the 1's complement (i.e., bitwise NOT) of the positive signal. In Ref. 11, the authors have extended the previous algorithm to handle up to eight transmit apertures and they showed that RCs outperform OSTBCs using IM/DD in uncorrelated log-normal channels. In Ref. 13, the authors show that coherent and differential OSTBCs in FSO systems outperform their counterpart RC systems at the expense of laborious receiver.

Previous studies have considered the case of uncorrelated channels assuming the spacing between transmitters is larger than the fading correlation length, but not exceeding the capture zone of the receiver, which should be less than $\theta L \mathrm{~m}$, where $\theta$ is the field of view of the receiver in radians (rad) and $L$ is the link distance in meter $(\mathrm{m}) .{ }^{12}$ For example, if $L=$ $1 \mathrm{~km}$ and $\theta \cong 10 \mathrm{mrad}$, then the transmitters should be $<10 \mathrm{~m}$ apart. However, the last assumption may be difficult in practice as available space for the transmitters may not be sufficient for this requirement. ${ }^{4}$

A simple and general method to approximate the probability density functions (PDF) of uncorrelated and correlated log-normal channels using moment generating function (MGF) is reported in Ref. 14. Another method for log-normal channels distribution approximation can be done using the Erlang distribution. ${ }^{15}$ The use of Erlang distribution leads to an approximate closed-form expression of the BER with log-normal channel variance not exceeding $3 \mathrm{~dB}$. Such variance is suitable for FSO communication systems. ${ }^{15}$ However, it is generally very difficult to find an optimal Erlang PDF for the employed log-normal channels.

Hence, the MGF approach is considered in this study to analyze FSO system performance employing RCs and OSTBCs using IM/DD in the presence of correlated lognormal channel conditions. Reported results demonstrate a 
close match between numerical Monte Carlo simulations and analytical results for a wide range of signal to noise ratio (SNR), which corroborates the exactness of our derivation.

The remainder of this paper is organized as follows: the FSO diversity model is presented in Sec. 2. In Sec. 3, performance analysis is presented. Numerical results and discussions are given in Sec. 4. Finally, conclusions are summarized in Sec. 5.

\section{System Model}

A synoptic diagram of the considered system model is depicted in Fig. 1. The system considers $N_{t}$ transmitters and a single receiver. Source bits are modulated using OOK and encoded by a multiple-input single-output (MISO) encoder. The encoder considers two techniques for transmission, namely OSTBCs and RCs. In RC systems, the same signal is transmitted simultaneously from the available transmitters, whereas in OSTBCs, full-rate real orthogonal matrices, $\mathbf{G}$, for $N_{t} \leq 8$, are considered. ${ }^{16}$ In Ref. 11, a linear transformation of $\mathbf{G}$ matrix to OOK matrix, $\mathbf{S}$, through $\mathbf{S}=$ $\frac{\mathbf{U}+\mathbf{G}}{2}$ is proposed, where $\mathbf{U}$ denotes a unit matrix with the same size as $\mathbf{G}$.

The encoded real symbols modulate the synchronized laser diodes. The transmitted light propagates over correlated log-normal channel and suffers from additive white Gaussian noise (AWGN) at the receiver input. As such, the received signal is given by

$r(t)=s(t) \eta \sum_{i=1}^{N_{t}} I_{i}+n(t)$,

where the received signal light intensity after turbulence, $I_{i}$ is given $b^{2}$

$I_{i}=I_{o} h_{i}$,

where $s(t)$ is the transmitted information symbol, $\eta$ is the optical to electrical conversion coefficient, $h_{i}=\exp \left(2 X_{i}\right)$ is the channel irradiance from transmitter $i$ to the receiver with $X_{i}$ being modeled as spatially correlated identically distributed Gaussian random variable with mean $\mu$, standard deviation $\sigma$ and variance $\sigma^{2}$. Hence, $h_{i}$ is a log-normal random variable (RV) with PDF given by ${ }^{17}$

$f\left(h_{i}\right)=\frac{1}{h_{i} \sqrt{8 \pi \sigma^{2}}} \times \exp \left(-\frac{\left(\ln \left(h_{i}\right)-2 \mu\right)^{2}}{8 \sigma^{2}}\right)$.

To ensure that the fading channel does not attenuate or amplify the average power, the fading coefficients are normalized as $\mathbf{E}\left[h_{i}\right]=e^{2\left(\mu+\sigma^{2}\right)}=1 . I_{o}$ in Eq. (2) is the received average signal light intensity without considering the channel effect and $n(t)$ is the AWGN at the receiver input. The noise is mainly due to shot noise which dominates

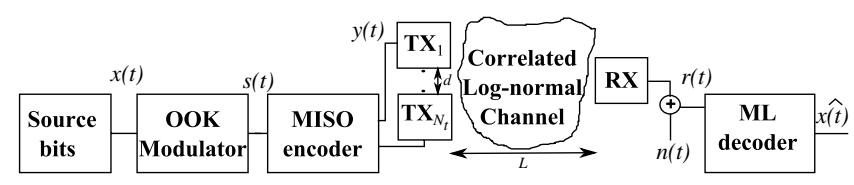

Fig. 1 Synoptic diagram of the proposed model. over other noise sources such as thermal, signal-dependent or dark noises. ${ }^{11}$

The spatial covariance matrix $\boldsymbol{\Gamma}$ coefficients, modeling the spatial correlation among transmitters, are given $b^{2}$

$\Gamma_{i j}=\sigma^{2} \times \rho^{|i-j|}$,

where $|$.$| stands for the absolute value, (\rho \leq 1)$ is the correlation coefficient, and $i$ and $j$ are the row and the column indices of the covariance matrix coefficients, respectively. According to Ref. 4, $\rho$ is a function of the separation distance $d$ between transmitters,

$\rho=\exp \left(-\frac{d}{d_{o}}\right)$

where the correlation length $d_{o} \approx \sqrt{\lambda L}$ with $\lambda$ being the wavelength and $L$ is the linear propagation distance between the transmitters and the receiver. This correlation model is called the exponential model ${ }^{18}$ and corresponds to the scenario of a multichannel transmission from linearly equispaced transmitters. From Eq. (5), the correlation of combined signals decays by increasing the spacing between the transmitters.

At the receiver side, maximum-likelihood decoder is considered to decode the received signals. ${ }^{16}$

\section{Performance Analysis}

\subsection{Orthogonal Space Time Block Codes}

The PDF of the instantaneous SNR $\gamma_{i}, 1 \leq i \leq N_{t}$, of correlated jointly log-normal channel is given by ${ }^{17}$

$f\left(\gamma_{1}, \cdots, \gamma_{N_{t}}\right)=\frac{\exp \left(-\frac{1}{32} Z(\boldsymbol{\Gamma})^{-1} Z^{T}\right)}{4^{N_{t}} \sqrt{(2 \pi)^{N_{t}}(\operatorname{det}[\boldsymbol{\Gamma}])} \Pi_{i=1}^{N_{t}} \gamma_{i}}$,

where $Z=\left[\ln \left(\frac{\gamma_{1}}{\bar{\gamma}_{1}}\right), \cdots, \ln \left(\frac{\gamma_{N_{t}}}{\bar{\gamma}_{t}}\right)\right]-4 \mu, \overline{\gamma_{i}}$ is the average SNR, $(\cdot)^{-1}$ is the inverse of a matrix, $(\cdot)^{T}$ is the transpose of a matrix, and det is the determinant of a matrix.

The instantaneous SNR $\gamma_{i}$ in Eq. (6) is given by ${ }^{19}$

$\gamma_{i}=\frac{\left(\eta I_{i}\right)^{2}}{N_{o}}$

The conditional BER for FSO system using OSTBCs is given by ${ }^{11}$

$P(e \mid h)=Q\left[\sqrt{\frac{E_{b}}{2 N_{o}} \sum_{i=1}^{N_{t}} h_{i}^{2}}\right]$,

with $E_{b}$ being the average electrical energy of the transmitted pulse at each transmitter given by ${ }^{11}$

$E_{b}=\left(\frac{\eta I_{o}}{N_{t}}\right)^{2}$

The factor $N_{t}$ is included in Eq. (9) to ensure that the total power of the considered MISO system is similar to the power of the benchmark single-input single-output (SISO) link. 
The alternative form of the Gaussian- $Q$ function is ${ }^{20}$

$$
\begin{aligned}
Q(y) & \triangleq \frac{1}{\sqrt{2 \pi}} \int_{y}^{\infty} \exp \left(-\frac{x^{2}}{2}\right) \mathrm{d} x \\
& =\frac{1}{\pi} \int_{0}^{\frac{\pi}{2}} \exp \left(-\frac{y^{2}}{2 \sin ^{2} \theta}\right) \mathrm{d} \theta .
\end{aligned}
$$

Substituting Eq. (9) into Eq. (8) and using Eq. (2) yields

$$
P(e \mid h)=Q\left(\sqrt{\frac{\eta^{2}}{2 N_{o}\left(N_{t}\right)^{2}} \sum_{i=1}^{N_{t}} I_{i}^{2}}\right) .
$$

Using Eq. (7), the conditional BER with respect to the SNR becomes

$$
P(e \mid h)=Q\left(\sqrt{\frac{\sum_{i=1}^{N_{t}} \gamma_{i}}{2\left(N_{t}\right)^{2}}}\right) .
$$

Using the PDF of the instantaneous SNR $\gamma_{i}$ in Eq. (6), the average bit error probability of OSTBCs is given by

$$
\mathrm{BER}=\int_{0}^{\infty} \cdots \int_{0}^{\infty} Q\left(\sqrt{\frac{\sum_{i=1}^{N_{t}} \gamma_{i}}{2\left(N_{t}\right)^{2}}}\right) f\left(\gamma_{1}, \cdots, \gamma_{N_{t}}\right) \mathrm{d} \gamma_{1} \cdots \mathrm{d} \gamma_{N_{t}} .
$$

According to Ref. 21, the MGF is defined by

$$
\Psi(s)=\int_{0}^{\infty} \cdots \int_{0}^{\infty} f\left(\gamma_{1}, \cdots, \gamma_{N_{t}}\right) \exp \left[-s \sum_{i=1}^{N_{t}} \gamma_{i}\right] \mathrm{d} \gamma_{1} \cdots \mathrm{d} \gamma_{N_{t}} .
$$

With the use of Eq. (6) and after few algebraic manipulations, the PDF of instantaneous branch SNR for the given branch $i, \gamma_{i}$, is

$f\left(\gamma_{i}\right)=\frac{1}{\sqrt{32 \pi \sigma^{2}} \gamma_{i}} \exp \left[-\frac{\left(\ln \left(\frac{\gamma_{i}}{\bar{\gamma}_{i}}\right)+4 \sigma^{2}\right)^{2}}{32 \sigma^{2}}\right]$.

The MGF of the RV $\gamma_{i}$ is then given by

$$
\begin{aligned}
\Psi(s)= & \int_{0}^{\infty} \exp \left(-s \gamma_{i}\right) \frac{1}{\sqrt{32 \pi \sigma^{2}} \gamma_{i}} \\
& \times \exp \left[-\frac{\left(\ln \left(\frac{\gamma_{i}}{\bar{\gamma}_{i}}\right)+4 \sigma^{2}\right)^{2}}{32 \sigma^{2}}\right] \mathrm{d} \gamma_{i} .
\end{aligned}
$$

Making the change of variable $x=\frac{\left(\ln \left(\frac{y_{i}}{\bar{\gamma}_{i}}\right)+4 \sigma^{2}\right)}{\sqrt{32 \sigma^{2}}}$ yields

$$
\Psi(s)=\frac{1}{\sqrt{\pi}} \int_{-\infty}^{\infty} \exp \left(-x^{2}\right) \exp \left[-s \bar{\gamma}_{i} \exp \left(\sqrt{32 \sigma^{2}} x-4 \sigma^{2}\right)\right] \mathrm{d} x
$$

Using Hermite polynomial approximation gives ${ }^{22}$

$$
\int_{-\infty}^{\infty} \exp \left(-x^{2}\right) g(x) \mathrm{d} x \approx \sum_{i=1}^{N} w_{i} g\left(x_{i}\right)
$$

where $w_{i}$ and $x_{i}$ are the weights and the roots of the Hermite polynomial, respectively.

Applying Eq. (18) on Eq. (17) yields

$\Psi(s) \approx \frac{1}{\sqrt{\pi}} \sum_{i=1}^{N} w_{i} \exp \left[-s \bar{\gamma}_{i} \exp \left(\sqrt{32 \sigma^{2}} x_{i}-4 \sigma^{2}\right)\right]$.

Using Eqs. (19), (6), and following a similar approach as in Ref. 14, the general form of the MGF in a correlated lognormal channel is given by

$$
\begin{aligned}
\Psi(s) \approx & \sum_{n_{1}=1}^{N} \cdots \sum_{n_{N_{t}}=1}^{N}\left[\prod_{i=1}^{N_{t}} \frac{w_{n_{i}}}{\sqrt{\pi}}\right] \\
& \times \exp \left(-s \sum_{i=1}^{N_{t}} \bar{\gamma}_{i}\left[\exp \left(\sqrt{32} \sum_{j=1}^{N_{t}} c_{i j}^{\prime} x_{n_{j}}-4 \sigma^{2}\right)\right]\right),
\end{aligned}
$$

with $N$ being the order of approximation of the Hermite polynomial. The values of $w_{n_{i}}$ and $x_{n j}$ of the $N$ 'th order Hermite polynomial are tabulated in Ref. 22 (Table 25.10), and $c_{k j}^{\prime}$ is the $(k, j)$ 'th coefficient of $\boldsymbol{\Gamma}_{\mathrm{sq}}=\boldsymbol{\Gamma}^{1 / 2}$.

Using Eq. (20) and assuming that all the average SNR from the transmitters are equal, i.e., $\bar{\gamma}_{i}=\bar{\gamma}, \forall i=$ $1, \cdots, N_{t}$, an approximate novel expression of the BER of OSTBCs over correlated log-normal channel, after some manipulations, is derived as

$$
\begin{aligned}
\mathrm{BER} \approx & \sum_{n_{1}=1}^{N} \ldots \sum_{n_{N_{t}}=1}^{N}\left[\prod_{i=1}^{N_{t}} \frac{w_{n_{i}}}{\sqrt{\pi}}\right] \\
& \times Q\left(\sqrt{\frac{\bar{\gamma}}{2 N_{t}^{2}} \sum_{i=1}^{N_{t}}\left[\exp \left(\sqrt{32} \sum_{j=1}^{N_{t}} c_{i j}^{\prime} x_{n_{j}}-4 \sigma^{2}\right)\right]}\right) .
\end{aligned}
$$

\subsection{Repetition Codes}

The conditional BER of FSO system using RCs is given by ${ }^{11}$

$P(e \mid h)=Q\left(\sqrt{\frac{E_{b}}{2 N_{o}}} \sum_{i=1}^{N_{t}} h_{i}\right)$

Using the alternative definition of the $Q$-function in Eq. (10), the BER of RC systems over correlated log-normal channel is given by

$$
\begin{aligned}
\operatorname{BER}= & \frac{1}{\pi} \int_{0}^{\infty} \cdots \int_{0}^{\infty} \int_{0}^{\frac{\pi}{2}} \exp \left(-\frac{\left(\sum_{i=1}^{N_{t}} \sqrt{\gamma_{i}}\right)^{2}}{4 N_{t}^{2} \sin ^{2} \theta}\right) \\
& \times f\left(\gamma_{1}, \cdots, \gamma_{N_{t}}\right) \mathrm{d} \theta \mathrm{d} \gamma_{1} \cdots \mathrm{d} \gamma_{N_{t}} .
\end{aligned}
$$

Let us note that the MGF approach cannot be used to represent Eq. (23), since it is directly proportional to $\left(\sqrt{\gamma_{i}}\right)^{2}$. However, under the condition that $\sigma^{2} \ll 1$, the following approximation can be used 
$I_{1}=\left(\sum_{i=1}^{N_{t}} \sqrt{\gamma_{i}}\right)^{2} \approx I_{2}=N_{t} \sum_{i=1}^{N_{t}} \gamma_{i}$

Monte Carlo simulation results with $10^{5}$ samples are conducted to verify the validity of the proposed approximation in Eq. (24). The relative estimation error percent, $\xi=$ $100 \times\left(\frac{I_{1}-I_{2}}{I_{1}}\right) \leq 8.2 \%$, is calculated for different $\sigma$ values. Let us note that the maximum value of $\sigma$ for log-normal channel corresponds to the maximum scintillation index (SI) value for log-normal channel, which is $\leq 0.75 .^{20}$ The relation between SI and $\sigma$ is defined in Ref. 17 as $\sigma=$ $\left(\frac{\sqrt{\ln (\mathrm{SI})+1}}{2}\right)$. Hence, $\sigma$ should be $\leq 0.374$. Estimation error percents for different values of $\sigma$ are tabulated in Table 1, where it is shown that the maximum error is $\leq 8.2 \%$.

Hence, substitute Eq. (24) in Eq. (23) and using Eq. (14) yields

$\mathrm{BER} \approx \frac{1}{\pi} \int_{0}^{\frac{\pi}{2}} \Psi\left(\frac{1}{4 N_{t} \sin ^{2} \theta}\right) \mathrm{d} \theta$.

From Eqs. (20) and (25) and assuming that all SNR averages from the transmitters are equal, an approximate novel expression for the BER of RC systems over correlated lognormal channels, after some manipulations, is derived as

$$
\begin{aligned}
\mathrm{BER} \approx & \sum_{n_{1}=1}^{N} \cdots \sum_{n_{N_{t}}=1}^{N}\left[\prod_{i=1}^{N_{t}} \frac{w_{n_{i}}}{\sqrt{\pi}}\right] \\
& \times Q\left(\sqrt{\frac{\bar{\gamma}}{2 N_{t}} \sum_{i=1}^{N_{t}}\left[\exp \left(\sqrt{32} \sum_{j=1}^{N_{t}} c_{i j}^{\prime} x_{n_{j}}-4 \sigma^{2}\right)\right]}\right)
\end{aligned}
$$

It is important to note that even though the derived expressions in Eqs. (21) and (26) are for correlated log-normal channels, they can be applied to uncorrelated log-normal channels by setting $\rho=0$ in $c_{i j}^{\prime}$.

\section{Numerical Results and Discussions}

In Fig. 2, the relation between the correlation effect and the separation distance between the transmit units is studied. The selected wavelength is $\lambda=1550 \mathrm{~nm}$ and different propagating distances and correlation lengths are considered. Simulation results show that if a correlation coefficient of $\rho \leq 10^{-3}$ is to be maintained, then the distance between

Table 1 Error due to approximation.

\begin{tabular}{lll}
$\sigma$ & $\rho$ & $\xi(\%)$ \\
\hline 0.1 & 0.5 & 0.4 \\
0.1 & 0.25 & 0.7 \\
0.374 & 0.5 & 5.9 \\
0.374 & 0.25 & 8.2 \\
\hline
\end{tabular}

the transmit units should be at least seven times the correlation length.

In Figs. 3-6, a target BER for the FSO system of $10^{-9}$ is assumed. ${ }^{19}$ The diversity branches are assumed to be identically distributed, and low or high correlation values are considered $(\rho=0.25$ and 0.5$)$.

In Fig. 3, correlated log-normal channel with $\sigma=0.1$ and $N_{t}=2$ is employed. Analytical results with $N=10$ are compared to Monte Carlo simulation results. Results demonstrate the close match for a wide range of SNR values. It is revealed that RCs are more power efficient than OSTBCs by about $3 \mathrm{~dB}$ for the two considered cases, $\rho=0.25$ and 0.5 .

Figures 4-6 study the performance of RCs and OSTBCs for different parameters $\sigma, \rho$, and $N_{t}$. In Fig. 4, the number of transmitters increases to three, $N_{t}=3$. It is shown that RCs with $N_{t}=3$ requires 0.6 and $0.5 \mathrm{~dB}$ less SNR for $\rho=0.25$ and 0.5 as compared to RCs with $N_{t}=2$, respectively. However, increasing the number of transmitters degrades the performance of OSTBCs by 1 and $1.3 \mathrm{~dB}$ for 0.25 and 0.5 , respectively. Performance degradation can be noticed when comparing the results in Fig. 3 with the results in Fig. 4.

In Figs. 5 and 6, a different log-normal parameter $\sigma=0.374$ with $N_{t}=2$ is employed. Figure 5 shows that increasing the atmospheric turbulence degrades the BER performance of the FSO system severely. The required SNR to achieve the target BER increases by 18.9 and $21 \mathrm{~dB}$ for RCs and by 19.3 and $21.5 \mathrm{~dB}$ for OSTBCs codes with $\rho=0.25$ and 0.5 , respectively, compared to $\sigma=0.1$ with $N_{t}=2$. Simulation results show that RCs still outperform OSTBCs by about $3 \mathrm{~dB}$.

Figure 6 shows results for $N_{t}=3$ and $\sigma=0.374$. An interesting observation is the behavior of OSTBCs results, where increasing the number of transmitters enhances the performance, unlike the reported behavior in Fig. 4. As compared to the results in Fig. 5, OSTBCs show an SNR gain of 1.95 and $2 \mathrm{~dB}$ for $\rho=0.25$ and 0.5 , respectively.

The behavior of OSTBC at low turbulence in FSO can be attributed to the fact that the channel paths from different transmitters to the receiver are highly correlated at low turbulence. In fact, if there is no turbulence, then the channel will be Gaussian and no diversity gain will be achieved. For Gaussian channels, the best system configuration is an SISO system, since in OSTBCs, the overall transmit power will be divided among the transmit antennas. Thereby, OSTBC systems with a low number of transmitters at low turbulence

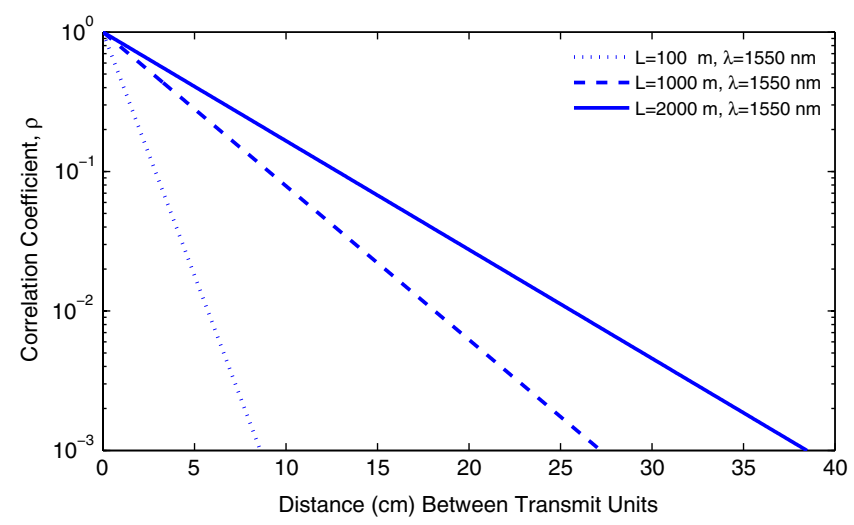

Fig. 2 Correlation effect versus distance between transmit units for difference propagation distances. 


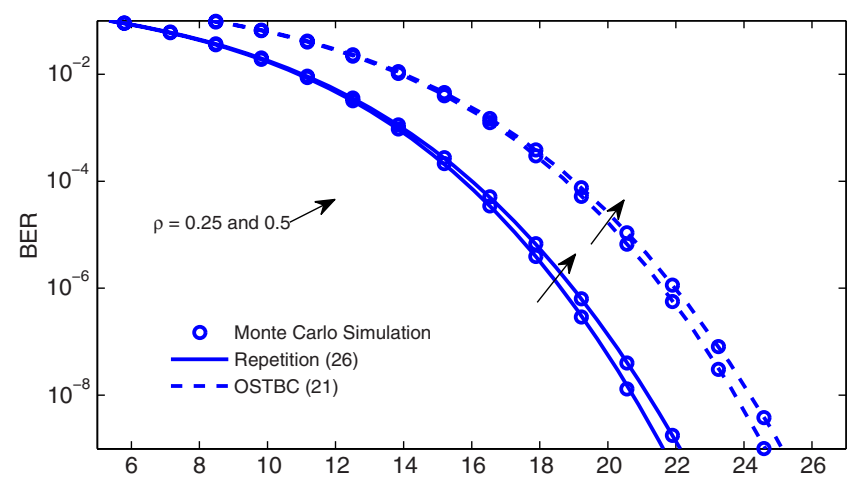

Avg. SNR[dB], Standard deviation $=0.1$, Number of transmitters $=2$

Fig. 3 Correlated log-normal with $\sigma=0.1, \mu=-\sigma^{2}$ and $N_{t}=2$.

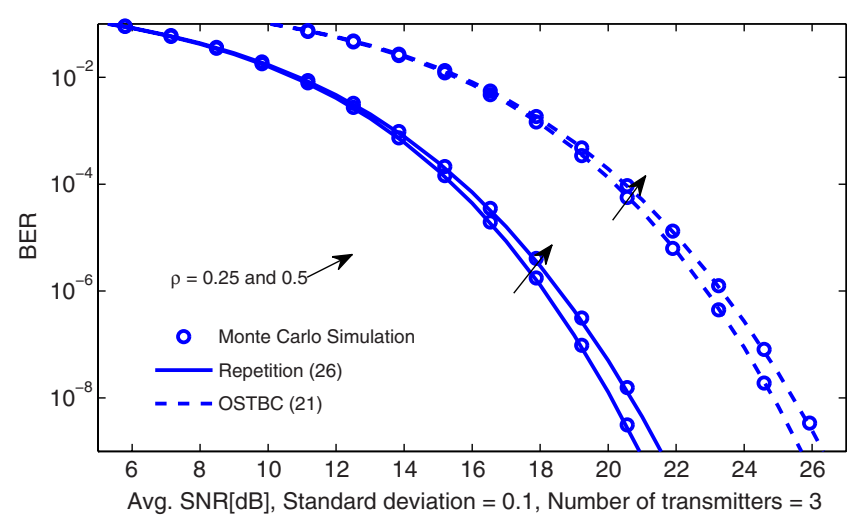

Fig. 4 Correlated log-normal with $\sigma=0.1, \mu=-\sigma^{2}$ and $N_{t}=3$.

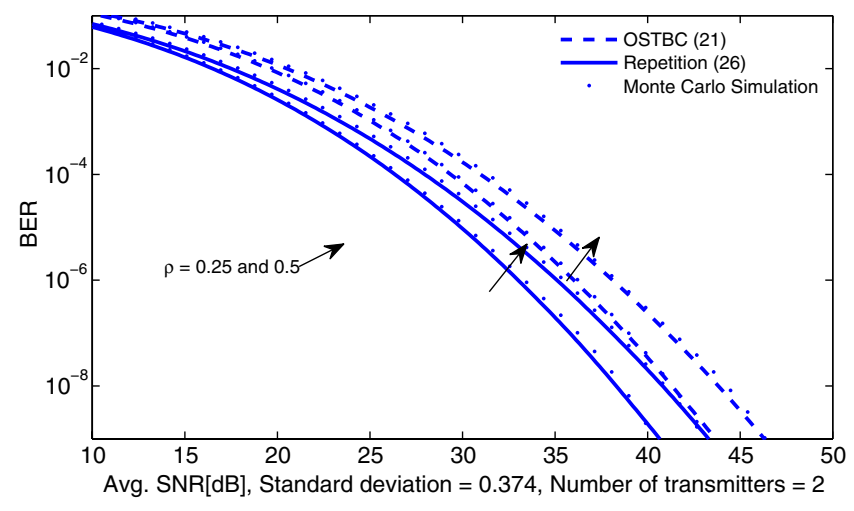

Fig. 5 Correlated log-normal with $\sigma=0.374, \mu=-\sigma^{2}$ and $N_{t}=2$.

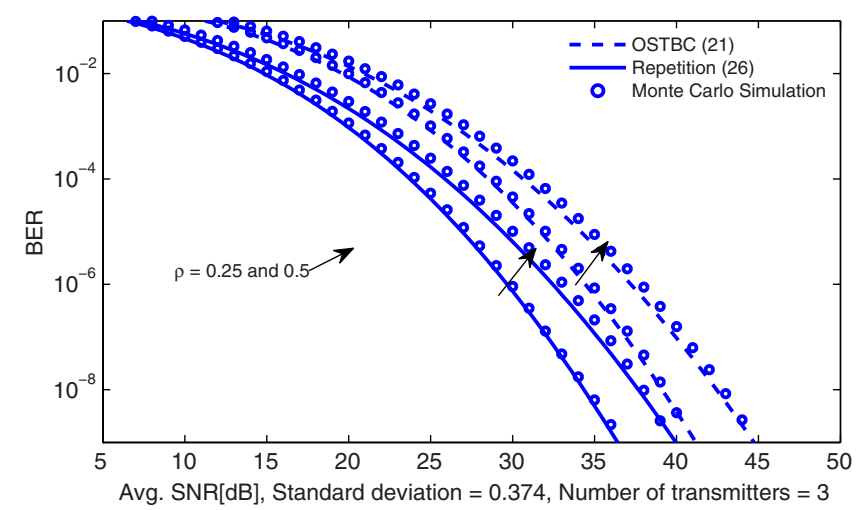

Fig. 6 Correlated log-normal with $\sigma=0.374, \mu=-\sigma^{2}$ and $N_{t}=3$. channel conditions outperform their counterpart systems with a higher number of transmitters.

The required SNR, under more severe turbulence, to achieve the target BER increases by 14.5 and $17.7 \mathrm{~dB}$ for RCs and 15.4 and $17.6 \mathrm{~dB}$ for OSTBCs with $\rho=0.25$ and 0.5 , respectively, compared to $\sigma=0.1$ with $N_{t}=3$.

\section{Conclusions}

The novel performance analysis of RCs and OSTBCs in an FSO system is investigated in this paper over correlated lognormal channels. Both schemes are shown to be useful in mitigating the turbulence fading effect and improving the BER performance. However, results revealed that RC systems always outperform their counterpart OSTBC systems. Furthermore, it is shown that the SNR gain of RC systems always increases with increasing the number of transmitters. On the contrary, increasing the number of transmitters of OSTBCs system does not always achieve higher SNR gain and the achieved gain depends on the channel conditions. Analytical and simulation results are shown to match over wide range of SNR and for different system parameters.

\section{Acknowledgments}

The authors gratefully acknowledge the support for this work from SNCS Research Center at University of Tabuk under the grant from the Ministry of Higher Education in Saudi Arabia.

\section{References}

1. E. Bayaki, R. Schober, and R. K. Mallik, "Performance analysis of free-space optical systems in Gamma-Gamma fading," in IEEE Conf. Global Communications, New Orleans, Louisiana, pp. 1-6, IEEE (2008).

2. S. M. Navidpour, M. Uysal, and M. Kavehrad, "BER performance of free-space optical transmission with spatial diversity," IEEE Trans. Wireless Commun. 6(8), 2813-2819 (2007).

3. M. R. Abaza et al., "MIMO techniques for high data rate free space optical communication system in log-normal channel," in Proc. Int. Conf. Technological Advances in Electrical, Electronics and Computer Engineering, Konya, Turkey, pp. 1-5, IEEE (2013).

4. X. Zhu and J. M. Kahn, "Maximum-likelihood spatial-diversity reception on correlated turbulent free-space optical channels," in IEEE Conf. Global Communications, Vol. 2, pp. 1237-1241, San Francisco, California (2000).

5. M. R. Abaza, N. A. Mohammed, and M. H. Aly, "BER performance of M-ary PPM free-space optical communications with channel fading," in Proc. 8th Int. Conf. High Capacity Optical Networks \& Enabling Technologies (HONET 2011), Riyadh, Saudi Arabia, pp. 111-115, IEEE (2011).

6. W. Popoola, Z. Ghassemlooy, and V. Ahmadi, "Performance of subcarrier modulated free-space optical communication link in negative exponential atmospheric turbulence environment," Int. J. Auton. Adapt. Commun. Syst. 1(3), 342-355 (2008).

7. A. Mansour, J. Youssef, and K. C. Yao, "Underdetermined BSS of MISO OSTBC signals," in Independent Component Analysis and Blind Source Separation, Paraty, Brazil, pp. 678-685, Springer (2009).

8. A. Maaref and S. Aissa, "Performance analysis of orthogonal space time block codes in spatially correlated MIMO Nakagami fading channels," IEEE Trans. Wireless Commun. 5(4), 807-817 (2006).

9. R. Mesleh et al., "Indoor MIMO optical wireless communication using spatial modulation," in IEEE Int. Conf. Communications, Cape Town, South Africa, pp. 1-5, IEEE (2010).

10. V. Choqueuse et al., "Blind channel estimation for STBC systems using higher-order statistics," IEEE Trans. Wireless Commun. 10(2), 495-505 (2011).

11. M. Safari and M. Uysal, "Do we really need OSTBC for free-space optical communication with direct detection?" IEEE Trans. Wireless Commun. 7(11), 4445-4448 (2008).

12. M. K. Simon and V. Vilnrotter, "Alamouti-type space-time coding for free-space optical communication with direct detection," IEEE Trans. Wireless Commun. 4(1), 35-39 (2005).

13. E. Bayaki and R. Schober, "Performance and design of coherent and differential space-time coded FSO systems," J. Lightw. Technol. 30(11), 1569-1577 (2012). 
14. N. B. Mehta et al., "Approximating the sum of correlated lognormal or lognormal-Rice random variables," IEEE Trans. Wireless Commun. 6(7), 2690-2699 (2007).

15. C. Abou-Rjeily and M. Bkassiny, "On the achievable diversity orders over non-severely faded log-normal channels," IEEE Commun. Lett. 14(8), 695-697 (2010).

16. V. Tarokh, H. Jafarkhani, and A. R. Calderbank, "Space-time block codes from orthogonal designs," IEEE Trans. Inf. Theory 45(5), 1456-1467 (1999)

17. H. Moradi, H. H. Refai, and P. G. LoPresti, "A switched diversity approach for multi-receiving optical wireless systems," Appl. Opt. 50(29), 5606-5614 (2011)

18. G. K. Karagiannidis, D. A. Zogas, and S. A. Kotsopoulos, "An efficient approach to multivariate Nakagami-m distribution using greens matrix approximation," IEEE Trans. Wireless Commun. 2(5), 883-889 (2003).

19. T. A. Tsiftsis et al., "Optical wireless links with spatial diversity over strong atmospheric turbulence channels," IEEE Trans. Wireless Commun. 8(2), 951-957 (2009).

20. K. Kiasaleh, "Performance of APD-based, PPM free-space optical communication systems in atmospheric turbulence," IEEE Trans. Commun. 53(9), 1455-1461 (2005).

21. H. Moradi, H. H. Refai, and P. G. LoPresti, "Circular MIMO FSO nodes with transmit selection and receive generalized selection diversity," IEEE Trans. Veh. Technol. 61(3), 1174-1181 (2012).

22. M. Abramowitz and I. A. Stegun, Handbook of Mathematical Functions with Formulas, Graphs, and Mathematical Tables, 9th ed., Dover Publications, New York (1972).

Mohamed Abaza received his BSc and MSc degrees in electronics and communications engineering from the Arab Academy for Science, Technology and Maritime Transport (AASTMT) (Cairo-Egypt) in 2009 and 2012, respectively. From 2009 to 2012, he was a teaching assistant in AASTMT. Since December 2012, he has been a PhD candidate at the Universite de Bretagne Occidentale UBO
(Brest, France). His current research interests include multipleinput multiple-output techniques and optical wireless communications.

Raed Mesleh holds a PhD in electrical engineering from Jacobs University in Bremen, Germany, and several years of postdoctoral wireless communication and optical wireless communication research experience in Germany. In October 2010, he joined University of Tabuk in Saudi Arabia, where he is now an assistant professor and the director of research excellence unit. His main research interests are in spatial modulation, MIMO cooperative wireless communication techniques, and optical wireless communication.

Ali Mansour received his MSc and PhD degrees in INPG, France, in July 1993 and January 1997, respectively, and his Habilitation a Diriger des Recherches on November 2006 from UBO, France. $\mathrm{He}$ had held six different positions where he was asked to conduct new research axes: TIRF-INPG Grenoble-France (speech enhancement), BMC-RIKEN Nagoya-Japan (robotics, biomedical signals), ENSIETA-Brest-France (COMINT, ELINT, PAT), Curtin UniversityPerth-Australia (wireless telecommunication, biomedical signals), Tabuk University KSA (telecommunication), and ENSTA-Bretagne Brest-France (cognitive radio).

El-Hadi M. Aggoune received his MS and $\mathrm{PhD}$ degrees in electrical engineering from the University of Washington (UW), Seattle, USA. $\mathrm{He}$ is listed as inventor in a major patent assigned to the Boeing Company. Currently, he is a professor and director of the Sensor Networks and Cellular Systems (SNCS) Research Center, University of Tabuk, Tabuk, Saudi Arabia. His research interests include modeling and simulation of large scale networks, sensor networks, and control and energy systems. 